\title{
Subtraction by addition
}

\author{
JAMIE I. D. CAMPBELL \\ University of Saskatchewan, Saskatoon, Saskatchewan, Canada
}

\begin{abstract}
University students' self-reports indicate that they often solve basic subtraction problems $(13-6=$ ?) by reference to the corresponding addition problem $(6+7=13$; therefore, $13-6=7)$. In this case, solution latency should be faster with subtraction problems presented in addition format $\left(6+_{-}=13\right)$ than in standard subtraction format $\left(13-6=\_\right)$. In Experiment 1 , the addition format resembled the standard layout for addition with the sum on the right $\left(6++_{-}=13\right)$, whereas in Experiment 2, the addition format resembled subtraction with the minuend on the left $(13=6+\ldots)$. Both experiments demonstrated a latency advantage for large problems (minuend $>10$ ) in the addition format as compared with the subtraction format $(13-6=$ _), although the effect was larger in Experiment 1 (254 msec) than in Experiment 2 (125 msec). Small subtractions (minuend $\leq 10)$ in Experiment 1 were solved equally quickly in the subtraction or addition format, but in Experiment 2, performance on small problems was faster in the standard format $(5-3=\ldots)$ than in the addition format $\left(5=3+_{-}\right)$. The results indicate that educated adults often use addition reference to solve large simple subtraction problems, but that they rely on direct memory retrieval for small subtractions.
\end{abstract}

The cognitive processes that subserve performance of elementary calculation (e.g., $2+6=8 ; 6 \times 7=42$ ) have received extensive experimental analysis over the last quarter century. The central goals have been to understand the representations, retrieval processes, and procedural strategies that subserve elementary math (Campbell, 2005). Until the mid-1990s, the prominent view was that educated adults relied predominantly on direct memory retrieval for simple arithmetic (Ashcraft, 1992; Campbell \& Graham, 1985). Young children's arithmetic, of course, is often based on counting or on other procedural strategies, but retrieval is evident even during preschool years, especially for small problems, such as $1+2$ (Siegler \& Shrager, 1984). It was commonly assumed that a gradual switch from procedural strategies to retrieval began during the early public school grades, and that by college age, most of the basic arithmetic facts presumably would be committed to memory (see Ashcraft, 1992, 1995; Campbell, 1995).

It has become increasingly apparent, however, that educated adults do not rely exclusively on direct retrieval for simple addition (Geary \& Wiley, 1991; LeFevre, Sadesky, \& Bisanz, 1996), multiplication (LeFevre, Bisanz, et al., 1996), division (Campbell, 1999; LeFevre \& Morris, 1999), or subtraction (Geary, Frensch, \& Wiley, 1993). Instead, across diverse cultural groups, adults often use procedural strategies based on counting and transformation for simple arithmetic. Such procedural strategies are most common on relatively large simple arithmetic problems $(6+9=15)$ and are very infrequent for smaller number problems $(2+3=5)$ (see, e.g., Campbell \& Xue, 2001; LeFevre, Sadesky, \& Bisanz, 1996). In the context of el- ementary arithmetic, small problems are often defined as those with either a sum/minuend $\leq 10$ (Seyler, Kirk, \& Ashcraft, 2003) or a product/dividend $\leq 25$ (Campbell \& Xue, 2001). Small arithmetic problems are encountered more frequently; consequently, the small problems are more likely to have high memory strength (see Zbrodoff \& Logan, 2005, for a discussion of factors contributing to the problem-size effect). The problem-size effect on strategy choice is also expressed in longer response times (RTs) and more errors for larger problems. This occurs, in part, because procedural strategies generally are slower and more error prone than direct retrieval. Nonetheless, when only trials reportedly solved by direct retrieval are analyzed, a substantial problem-size effect remains both for RT and for errors (Campbell, Fuchs-Lacelle, \& Phenix, 2006; Campbell \& Xue, 2001).

The use of procedural strategies for simple arithmetic also varies across arithmetic operations. For example, Campbell and Xue (2001) found that non-Asian Canadian students reported using procedures $43 \%$ of the time for simple division, $42 \%$ for subtraction, $24 \%$ for addition, but only $4 \%$ for multiplication. Estimates for the use of procedural strategies vary considerably across studies (e.g., reported procedure use for simple multiplication by North American university students was $13 \%$ in Campbell \& Timm, 2000; 22\% in Hecht, 1999; 15\% in LeFevre, Bisanz, et al., 1996; and 41\% in LeFevre \& Morris, 1999), but it is generally found that procedure use is more common for simple subtraction and division than for the inverse operations of addition and multiplication. In part, this asymmetry in procedure use is an outcome of educational practice. Addition and multiplication typically are

J. I. D. Campbell, jamie.campbell@usask.ca 
learned first as the basic operations, whereas subtraction and division are introduced later and are taught in relation to the inverse operations. Another factor is the relative difficulty of executing procedural strategies across operations. For example, a counting strategy for simple addition $(8+3=8,9,10,11)$ is relatively easy and efficient, but counting for multiplication entails repeated additions $(8 \times$ $3=8,16,24)$ and is therefore relatively difficult. This difference probably explains why procedural strategies usually are reported less often for multiplication than for addition (see Campbell \& Xue, 2001). Similarly, strategies based on the knowledge of the inverse relation between addition and subtraction (e.g., $4+3=7$ corresponds to $7-3=4$ ) and between multiplication and division (e.g., $4 \times 9=36$ corresponds to $36 \div 9=4$ ) permit skill in one operation to mediate performance on the complementary operation. Such mediation probably begins early in the development of arithmetic skills (Barrouillet, Mignon, \& Thevenot, 2008; Robinson, 2001) and may become very efficient by adulthood (Campbell, 1997, 1999). People may never develop direct memory for all the basic subtraction and division problems because of the availability of efficient procedural methods.

Division mediated by multiplication may be especially afforded by features of the memory representations that develop for multiplication facts. Rickard (2005) proposed that multiplication fact representations include a reverse association that provides direct access to factors (e.g., 7 and 5) given presentation of their product (e.g., 35; see also Rusconi, Galfano, Rebonato, \& Umiltà, 2006). Rickard demonstrated that university students are efficient at factoring (i.e., stating the factors given the product) and that factoring transfers to the corresponding multiplication fact (see also Campbell \& Robert, 2008). When direct retrieval of a division fact is not possible, efficient division by factoring would be possible via the reverse association. For example, the presentation of $56 \div 7$ would activate the reverse association from 56 to 7 and 8 , with the unique element (8) reported as the quotient. Given that multiplication retrieval is faster than division retrieval, division by factoring could explain the results of Mauro, LeFevre, and Morris (2003), who found faster times to answer large division problems presented in a multiplication format (e.g., $8 \times \times_{-}=56$ ) rather than in a division format $(56 \div 8=\ldots)$.

Rickard (2005) pointed out that a reverse association would develop for multiplication facts, but that it would not develop for addition facts. This difference would arise because most products of single-digit numbers are associated with a unique pair of factors (e.g., 27 with 3 and 9), but sums do not correspond to a unique pair of addends. Consequently, practicing multiplication would establish both forward and reverse associations, but practicing addition would not. Without a reverse addition association to mediate subtraction, it seems to follow that addition-based mediational strategies should be relatively infrequent for subtraction. There is a performance diagnostic for mediation, because mediation strategies give rise to positive transfer between corresponding problems in the inverse operation (e.g., practicing $8 \times 7=56$ facilitates perfor- mance of $56 \div 7=8$; Campbell, 1999; Campbell et al., 2006; LeFevre \& Morris, 1999). Strengthening the mediator produces RT savings because the speed of mediation depends on the accessibility of the mediator. To test for transfer between addition and subtraction, Campbell et al. examined prime-probe problem pairs within the same block of trials. There was no evidence for positive RT transfer from subtraction to addition (e.g., prime $15-9$, probe $6+9$ ) or from addition to subtraction (prime $6+9$, probe $15-6$ ). Thus, there was no evidence of mediation of subtraction by addition.

Nonetheless, other research seems to contradict this conclusion. Several studies have found that adults report solving subtractions by reference to the corresponding addition fact (e.g., $15-6$ is 9 because $6+9=15$ ) (Geary et al., 1993; LeFevre, DeStefano, Penner-Wilger, \& Daley, 2006; Seyler et al., 2003). Furthermore, transformation strategies - such as addition reference-are especially common for the larger subtractions with a minuend greater than 10 (e.g., $11-6=5$ ). Campbell and Xue (2001) found that, on average, Canadian university students reported transformation strategies for $44 \%$ of large subtraction problems, whereas transformation was infrequent for small subtractions (14\%). LeFevre et al. (2006) similarly estimated transformation strategies for large subtraction problems to be approximately $40 \%$, as compared with only $10 \%$ for small subtractions in their sample of Canadian university students. Thus, the evidence is mixed for mediation of subtraction by addition, with participants' self-reports suggesting that such transformation strategies are common for large subtractions, whereas Campbell et al. (2006) found no evidence for the positive transfer that would be expected if mediation was common.

\section{The Present Experiments}

To seek objective evidence for mediation of subtraction via addition, the following experiments used the procedure developed by Mauro et al. (2003) for division and multiplication and applied it to addition and subtraction. Mauro et al. based their procedure on the results of LeFevre and Morris (1999), who found that university students reported recasting division problems into the corresponding multiplication problem (e.g., converting $56 \div 8$ into $8 \times x_{-}=56$ ) on about $45 \%$ of trials. Recasting of division as multiplication was more likely for large than for small division problems, which were predominantly solved by direct retrieval. Given this likelihood, they reasoned that presenting problems in the multiplication format (e.g., $8 \times_{-}=56$ ) should result in faster RTs than the standard division format (e.g., $72 \div 8=$ ), particularly for the large division problems that are most likely to be recast as multiplication. RT savings would occur because presenting the division problem in multiplication format would save having to mentally rearrange problem elements into multiplication format. Additionally, the multiplication format probably presents a better retrieval cue for the corresponding multiplication problem than does the standard division format. Their results confirmed a 66-msec RT advantage for division in multiplication format, and this was observed selectively for the large division problems and 
not for small division problems. In contrast, multiplication problems recast as division $(-\div 3=9)$ were extremely slow and error prone because of a tendency to perform the wrong operation (e.g., responding "three" to $\_3=9$ ). Their results support the conclusion that large division problems often are solved by conversion to multiplication but that the reverse is not true.

In the present article, the same reasoning was extended to addition and subtraction. If large subtraction problems (e.g., $11-6=$ _) are often mediated by the corresponding addition problems, then the subtraction RT should be faster in the addition format $\left(6++_{-}=11\right)$. In contrast, if small subtractions (i.e., minuend $\leq 10$ ) are primarily solved by direct retrieval, then there should be no RT advantage for subtraction in addition format for small subtractions. Similarly, so-called tie problems (e.g., $6-3=3 ; 16-8=8$ ), which are consistently found to be solved by direct retrieval (see, e.g., Campbell \& Gunter, 2002), should present no RT advantage when recast as addition. Finally, by analogy to the results of Mauro et al. (2003) for division, performance on addition problems recast as subtraction (e.g., $--6=5$ ) would be expected to be very slow and prone to operation errors.

\section{EXPERIMENT 1}

\section{Method}

\section{Participants}

Ten women and 4 men, ranging in age from 18 to 28 years (mean $=$ 20) , participated to fulfill a research-credit option in their introductory psychology course at the University of Saskatchewan. Participants received one bonus mark toward their final course grade. The experiment was advertised as a study of simple arithmetic skills. Ten participants reported English as their first language for arithmetic, and 1 each reported Arabic, Chinese, French, and Hungarian. All participants answered problems in English.

\section{Apparatus}

Instructions and stimuli appeared on two high-resolution monitors displayed by a PC. The experimenter viewed one monitor, and the participant viewed the other. Participants sat approximately $50 \mathrm{~cm}$ from the monitor and wore a lapel microphone connected to a sound-activated relay that controlled a software clock accurate to $\pm 1 \mathrm{msec}$.

\section{Stimuli}

To understand the experimental stimuli, it is important that one distinguish between arithmetic operation and arithmetic format. The subtraction operation is defined by the formula minuend $(c) \pm$ subtrahend $(b)=$ difference $(a)$. Therefore, a problem that presents $c$ and $b$ and requires answer $a$ is, by definition, a subtraction problem. Thus, both $13-6={ }_{-}$and $6+\ldots=13$ are subtraction problems, but the former is a subtraction problem in subtraction format (henceforth denoted $\mathrm{S}-$ ), whereas the latter is subtraction in addition format (denoted $\mathrm{S}+$ ). Similarly, addition is defined by the formula augend $(a)+$ addend $(b)=\operatorname{sum}(c)$. A problem that presents $a$ and $b$ and requires answer $c$ is therefore an addition problem. Consequently, $7+6={ }_{-}$and -$6=7$ are both addition problems. The former is addition in addition format (denoted $\mathrm{A}+$ ), and the latter is addition in subtraction format $(\mathrm{A}-)$. The subtraction and addition stimuli were constructed as follows: There are 36 possible pairings of the Arabic digits 2 through 9 when operand order is ignored (e.g., $3+8$ vs. $8+3$ ), including eight ties (e.g., $8+8,2+2$ ). For each participant, one operand order or the other for the 28 nontie pairs was selected at random. Each of the 36 pairs provided the basis for addition and subtraction problems that par- alleled the six formats used for multiplication and division by Mauro et al. (2003). There were two formats of addition problems, including an addition format (A+; e.g., $7+6=\ldots$ ) and a subtraction format (A-; e.g., $-6=7$ ). The four formats used for subtraction problems included two subtraction formats $(\mathrm{S}-$; e.g., $13-6=, 13-$ $=6$ ) and two addition formats (S+; e.g., $6+{ }_{-}=13,{ }_{-}+\overline{6}=13$ ). Stimuli appeared horizontally as white characters against a dark background. Each stimulus was 9 or 10 characters wide, with character spaces approximately $3 \mathrm{~mm}$ wide and $5 \mathrm{~mm}$ high.

\section{Design}

Each operand pair was tested once in each of six trial blocks, and there were six trials involving each of the six formats within a block. Each operand pair was tested in all six formats across blocks. Thirty-six different orders of the six formats were constructed, and these were randomly assigned to operand pairs for each participant. To counterbalance order effects, each possible pairwise combination of successive format conditions across blocks occurred equally often among the 36 orders. The condition orders were 123456, 234561, 345612, 456123, 561234, 612345, 654321, 165432, 216543, $321654,432165,543216,132546,315264,256413,524631$, 461352, 643125, 645231, 462513, 314652, 136425, 253164, $521346,514263,142635,426351,263514,635142,351426$, $362415,536241,153624,415362,241536,624153$. Problem order within blocks was independently randomized for each participant.

\section{Procedure}

Testing took place in a quiet room with an experimenter present. Instructions described the experiment as a test of speed of basic numerical skills. The task was to state the correct answer to each problem as quickly as possible. Participants were advised that occasional errors are normal with speeded responding. The experimenter initiated each block of trials. Prior to each trial, a fixation dot appeared at the center of the screen for $1 \mathrm{sec}$, and then it flashed twice over a 1-sec interval. The stimulus appeared at the center of the screen on what would have been the third flash. Timing began when the stimulus appeared, and it ended when the response triggered the sound-activated relay. When a sound was detected, the problem display was cleared immediately, which allowed the experimenter to note failures of the voice-activated relay. Participants could take a brief rest between blocks, and the entire session required about $25 \mathrm{~min}$. Prior to the six blocks of experimental trials, there were 36 randomized practice trials, with six problems in each format using operand pairs not used for experimental trials (the digit 1 paired randomly with the digits 2-9).

\section{Results}

A total of 403 RTs (13.4\%) were excluded because of incorrect responses or microphone failures, or because they were discarded as outliers more than 2.5 standard deviations from a participant's cell means. Nontie problems with a sum or minuend $\leq 10$ were classified as small problems $(n=12)$; otherwise a nontie problem was classified as large $(n=16)$ (cf. LeFevre et al., 2006; Seyler et al., 2003).

\section{Subtraction Problems}

RT. Mean RT for correct subtraction trials (see Table 1) received a $2 \times 2 \times 3$ ANOVA with factors of format (subtraction, addition), problem type (ties, small, large), and appearance (standard, unusual). The standard arrangement for $\mathrm{S}-$ problems would be, for example, $13-6={ }_{-}$as compared with the more unusual $13-{ }_{-}=6$. The $\mathrm{S}+$ problems with the first operand missing were classified as unusual (e.g., _ $+6=13$ ), and those with the second operand missing were classified as standard $(6+\ldots=13)$. This classifi- 
Table 1

\begin{tabular}{|c|c|c|c|c|c|c|c|c|c|}
\hline \multirow{2}{*}{$\begin{array}{c}\text { Format } \\
\text { Example }\end{array}$} & \multicolumn{3}{|c|}{ RT } & \multicolumn{3}{|c|}{$\%$ Error } & \multicolumn{3}{|c|}{ \% Operation Errors } \\
\hline & Ties & Small & Large & Ties & Small & Large & Ties & Small & Large \\
\hline \multicolumn{10}{|c|}{ Subtraction Problems } \\
\hline $13-6={ }_{-}^{a}$ & 1,170 & 1,340 & 2,290 & 3 & 3 & 13 & 33 & 0 & 3 \\
\hline $6++_{-}=13^{\mathrm{a}}$ & 1,312 & 1,328 & 2,164 & 3 & 2 & 11 & 0 & 33 & 0 \\
\hline $13-{ }_{-}=6^{\mathrm{b}}$ & 1,400 & 1,564 & 2,599 & 4 & 6 & 17 & 0 & 20 & 3 \\
\hline$-+\overline{6}=13^{\mathrm{b}}$ & 1,311 & 1,572 & 2,217 & 5 & 10 & 12 & 50 & 50 & 15 \\
\hline \multicolumn{10}{|c|}{ Addition Problems } \\
\hline $7+6=$ & 935 & 1,058 & 1,614 & 0 & 2 & 10 & 0 & 33 & 14 \\
\hline$--6=7$ & 1,670 & 2,103 & 3,060 & 17 & 10 & 25 & 90 & 69 & 20 \\
\hline
\end{tabular}

cation followed that of Mauro et al. (2003) on the basis of their participants' self-reports about the unusualness of the corresponding division-as-multiplication problems (e.g., $\times 6=42$ seemed more unusual than $6 \times_{-}=42$ ). The main effect of appearance reflected faster mean RT with problems in standard format $(1,601 \mathrm{msec})$ than with those in unusual format $(1,777 \mathrm{msec})\left[F(1,13)=11.6, M S_{\mathrm{e}}=112,989, p=\right.$ $.005]$. The only other significant tests in the ANOVA were the main effect of type (ties, 1,298 msec; small, 1,452 msec; large, $2,317 \mathrm{msec})\left[F(2,26)=27.9, M S_{\mathrm{e}}=605,514, p<\right.$ $.001]$ and the format $\times$ type interaction $[F(2,26)=7.1$, $\left.M S_{\mathrm{e}}=46,999, p=.003\right]$, which is depicted in Figure 1. The figure shows that for large subtractions, RTs were faster for $\mathrm{S}+$ problems (e.g., $6+_{-}=13$ or ${ }_{-}+6=13$ ) than for $\mathrm{S}-$ problems (e.g., $13-6=\ldots$ or $13-_{-}=6$ ), but this effect was not observed for tie or small problems. There was no evidence for a three-way interaction $[F(2,26)<1$, $\left.M S_{\mathrm{e}}=101,270\right]$. A separate ANOVA of the large problems confirmed a robust main effect of format $[F(1,13)=12.8$, $\left.M S_{\mathrm{e}}=70,230, p=.003\right]$ owing to a $254-\mathrm{msec} \mathrm{RT}$ advantage for large $\mathrm{S}+(2,190 \mathrm{msec})$ as compared with small S$(2,444 \mathrm{msec})$. As Figure 1 suggests, tie and small problems did not present this effect $\left[F(1,13)<1, M S_{\mathrm{e}}=64,680\right]$. Thus, only the large subtraction problems presented evidence for an RT advantage in the addition format, as compared with the subtraction format.

Percentage of errors. The corresponding analysis of subtraction error rates (see Table 1) indicated only a main effect of problem type $\left[F(2,26)=16.2, M S_{\mathrm{e}}=94.5, p<\right.$ $.001]$ and of appearance $\left[F(1,13)=10.2, M S_{\mathrm{e}}=42.1\right.$, $p=.007]$, and these effects presented the same pattern as that in the RT analysis. Errors rates for tie, small, and large subtraction problems, respectively, were $3.6 \%, 5.1 \%$, and $13.3 \%$. Subtraction errors occurred less often with problems in standard appearance $(5.7 \%)$ than with those in unusual appearance $(8.9 \%)$. There were no other significant effects in the analysis of subtraction errors (all $p \mathrm{~s}>.2$ ).

\section{Addition Problems}

RT. Mean RT for correct addition trials (see Table 1) received a $2 \times 3$ ANOVA with factors of format (subtraction, addition) and problem type (ties, small, large). The experiment produced the standard effects of addition problem type (see, e.g., Campbell \& Xue, 2001), with ties yielding the fastest mean RT $(1,303 \mathrm{msec})$, followed by small $(1,580 \mathrm{msec})$ and large $(2,337 \mathrm{msec})$ problems $\left[F(2,26)=27.8, M S_{\mathrm{e}}=288,529, p<.001\right]$. Addition performance was much faster in the $\mathrm{A}+$ format $(1,203 \mathrm{msec})$ than in the $\mathrm{A}-$ format $(2,337 \mathrm{msec})[F(1,13)=76.1$, $\left.M S_{\mathrm{e}}=318,796, p<.001\right]$, but this varied across problem type, with the $\mathrm{A}+$ advantage greatest for large problems $(1,446 \mathrm{msec})$, followed by small $(1,045 \mathrm{msec})$ and then tie $(735 \mathrm{msec})$ problems $\left[F(2,26)=14.1, M S_{\mathrm{e}}=63,070\right.$, $p<.001]$. A comparison of the mean RTs for addition and subtraction problems in Table 1 shows that for all three problem types (i.e., tie, small, and large problems), A + problems (e.g., $7+6=$ _) were substantially faster on average (at least $235 \mathrm{msec}$ ) than any other condition, whereas A- problems (e.g., _ $-6=7$ ) were substantially slower (at least $270 \mathrm{msec}$ ) than any other condition. Thus, addition was faster than subtraction, except when addition problems were recast in the unfamiliar subtraction format. As is shown in the following analysis of addition errors, participants quite often performed subtraction (e.g., respond "one" to _ $-6=7$ ) instead of performing addition for these problems. Thus, participants had substantial

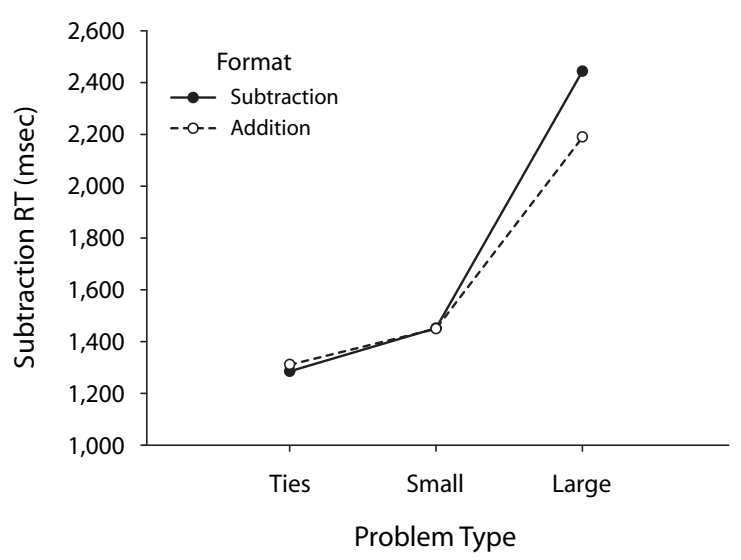

Figure 1. Mean subtraction response time (RT) as a function of problem type and format in Experiment 1. 
difficulty interpreting the $\mathrm{A}-$ problems, and this would contribute to their long RTs.

Percentage of errors. The corresponding analysis of addition errors (see Table 1) indicated only a main effect of problem type $\left[F(2,26)=5.1, M S_{\mathrm{e}}=207.4, p=\right.$ $.01]$ and of format $\left[F(1,13)=17.2, M S_{\mathrm{e}}=216.3, p<\right.$ $.001]$. Error rates for tie, small, and large addition problems, respectively, were $8.6 \%, 5.7 \%$, and $17.4 \%$. Addition errors occurred less often with problems in addition format $(3.9 \%)$ than with those in subtraction format $(17.2 \%)$. As Table 1 shows, there was a high proportion of operation errors for A- problems (e.g., responding "two" given _ $-3=5$ ), but a relatively small proportion of operation errors with A + format (responding "two" given $3+5=\ldots$ ). Participants evidently found the $\mathrm{A}-$ format difficult to interpret as addition, and they were sometimes lured by these stimuli to perform addition, resulting in an operation error. In general, the proportion of operation errors was higher for tie and small problems than for large problems. This result replicates previous research (Campbell, 1995) and likely occurred because the retrieval strength for tie and small problems is high, which makes them strong competitors for retrieval in the context of mixed addition and subtraction trials.

\section{Discussion}

Experiment 1 provided evidence that the mean RT to solve large subtraction problems was substantially faster in the addition format than in the subtraction format. This advantage was not observed either for small or for tie subtraction problems. The finding that large subtractions were facilitated by the $\mathrm{S}+$ format is consistent with university students' self-reported use of addition-based strategies for large subtractions (LeFevre et al., 2006; Seyler et al., 2003). The S+ format would facilitate this process by reducing the internal manipulations required to recast the problem as addition. In contrast, the $\mathrm{A}-$ format (e.g., _ $-6=7$ ) was extremely slow and error prone as compared with the A+ $(6+7=\ldots)$. Since two thirds of trials were subtraction problems, there would be a bias to expect subtraction. This, combined with presentation of the minus sign, would make A - problems very strong subtraction lures, as evidenced by the high proportion of operation errors made on $\mathrm{A}$ - problems.

The mean RT was $176 \mathrm{msec}$ faster with subtraction problems in standard format (e.g., $13-6={ }_{-}$or $6+_{-}=$ 13) as compared with unusual format $\left(13^{-}{ }_{-}=\overline{6}\right.$ or $+6=13)$. This result suggests that the unusual layouts interfered with processing of the problem. The appearance manipulation for the $\mathrm{S}+$ formats is somewhat ambiguous, however. Following Mauro et al. (2003), problems with the first operand missing were classified as unusual (e.g., - $+6=13$ ), and those with the second operand missing were classified as standard $\left(6++_{-}=13\right)$. Their rationale for these classifications was based on participants' self-reports about the unusualness of the corresponding division-as-multiplication problems (e.g., $\_\times 6=42$ seemed more unusual than $6 x_{-}=42$ ). There is no evidence, however, that participants here would have the same perception about the corresponding subtraction problems (e.g., ${ }_{-}+6=13$ vs. $6+_{-}=13$ ). To eliminate this ambiguity, the $\mathrm{S}+$ formats were rearranged for Experiment 2 so that the positions of the missing elements matched the corresponding subtraction problems in subtraction format. The rearrangement simply entailed moving the minuend from the right- to the left-hand side of the equation (see examples in Table 2). Doing this operationalized the appearance factor in the position of the missing element. A second consequence of the rearrangement was that the $\mathrm{S}+$ format now resembled the standard subtraction format with the minuend on the left, but this was an unusual arrangement for addition (e.g., $13=6+7$ ), which normally appears with the sum on the right $(6+7=13)$. If the advantage for $\mathrm{S}+$ format in Experiment 1 depended on the problem resembling the standard addition format $\left(6+{ }_{-}=13\right)$, then the effect would have been reduced or eliminated when rearranged to resemble subtraction $(13=6+\ldots)$, as in Experiment 2.

Finally, in Experiment 1, the A- format was the slowest and most error-prone condition, possibly because these problems mimicked standard subtraction with the implied minuend on the left (e.g., _- $6=7$ ). For Experiment 2, the $\mathrm{A}$ - format was rearranged by moving the difference from the right to the left (see Table 2) so that the location of the implied minuend did not mimic standard subtraction $(7=-6)$. If the difficulty of addition in subtraction format in Experiment 1 was due to the format's mimicking the layout of standard subtraction, then performance on A - problems in Experiment 2 should improve, as compared with that on standard $\mathrm{A}+$ format.

\section{EXPERIMENT 2}

\section{Method}

Eight women and 6 men $18-31$ years of age $($ mean $=21)$ were recruited in the same manner as in Experiment 1. Thirteen participants reported English as their first language for arithmetic, and 1 reported French. Experiment 2 was identical to Experiment 1, except that in three of the stimulus formats, the sum/minuend was relocated from the right side to the left side (i.e., $7={ }_{-}-6,13=6+_{-}$, $13=\ldots+6)$, whereas the other three formats remained the same as in Experiment $1\left(7+6=_{-}, 13-6=\ldots, 13-_{-}=6\right)$.

\section{Results}

A total of $400 \mathrm{RTs}$ (13.2\%) were excluded as outliers for the same reasons as in Experiment 1, or because of incorrect responses or microphone failures.

\section{Subtraction Problems}

RT. Mean correct RT for subtraction problems (see Table 2) received a $3 \times 2 \times 2$ ANOVA with factors of problem type (ties, small, large), format (subtraction, addition), and appearance (missing element center or right). The missing element on the right corresponded to standard subtraction format $(13-6=\ldots)$ or resembled it $(13=6+\ldots)$, and this facilitated subtraction RT by $122 \mathrm{msec}$, as compared with the missing element in the center $\left(13-_{-}=6\right.$ or $\left.13=_{-}+6\right)[F(1,13)=14.7$, $\left.M S_{\mathrm{e}}=42,956, p=.002\right]$. The main effect of type (ties, $1,186 \mathrm{msec}$; small, $1,320 \mathrm{msec}$; large, $1,728 \mathrm{msec}$ ) $\left[F(2,26)=12.4, M S_{\mathrm{e}}=360,913, p<.001\right]$ was qualified 
Table 2

\begin{tabular}{|c|c|c|c|c|c|c|c|c|c|}
\hline \multirow{2}{*}{$\begin{array}{c}\text { Format } \\
\text { Example }\end{array}$} & \multicolumn{3}{|c|}{ RT } & \multicolumn{3}{|c|}{$\%$ Error } & \multicolumn{3}{|c|}{$\%$ Operation Errors } \\
\hline & Ties & Small & Large & Ties & Small & Large & Ties & Small & Large \\
\hline \multicolumn{10}{|c|}{ Subtraction Problems } \\
\hline $13-6=$ & 1,171 & 1,139 & 1,702 & 4 & 1 & 6 & 0 & 0 & 0 \\
\hline $13=6+$ & 1,147 & 1,329 & 1,613 & 4 & 2 & 10 & 0 & 25 & 13 \\
\hline $13-{ }_{-}=\overline{6}$ & 1,269 & 1,408 & 1,880 & 3 & 4 & 11 & 33 & 33 & 4 \\
\hline $13=-+6$ & 1,156 & 1,406 & 1,718 & 4 & 3 & 13 & 40 & 60 & 14 \\
\hline \multicolumn{10}{|c|}{ Addition Problems } \\
\hline $7+6=$ & 878 & 1,001 & 1,355 & 0 & 3 & 8 & 0 & 20 & 6 \\
\hline $7=\ldots-\overline{6}$ & 1,668 & 2,057 & 2,666 & 12 & 12 & 27 & 62 & 65 & 41 \\
\hline
\end{tabular}

by the format $\times$ type interaction $\left[F(2,26)=11.0, M S_{\mathrm{e}}=\right.$ $16,631, p<.001]$ (see Figure 2), which was decomposed with separate format $\times$ appearance ANOVAs for each problem type.

The analysis of large subtraction problems confirmed a robust main effect of format $\left[F(1,13)=7.9, M S_{\mathrm{e}}=\right.$ $27,883, p=.015]$, reflecting a $125-\mathrm{msec} \mathrm{RT}$ advantage for large S+ problems $(1,666 \mathrm{msec})$, as compared with the $\mathrm{S}$ - format $(1,791 \mathrm{msec})$. The direction of the format effect on large problems replicated Experiment 1, but the size of the effect was half as large as it was in Experiment $1(254 \mathrm{msec})$. The $141-\mathrm{msec}$ effect of appearance was also significant $\left[F(1,13)=5.5, M S_{\mathrm{e}}=50,910, p=\right.$ $.04]$ for large problems, but there was no evidence for a format $\times$ appearance interaction $\left[F(1,13)<1, M S_{\mathrm{e}}=\right.$ $120,946]$.

The corresponding analysis of small problems indicated that small subtractions also were faster when the layout resembled standard subtraction with the missing element on the right $(1,234 \mathrm{msec})$ rather than in the center $(1,407 \mathrm{msec})$ $\left[F(1,13)=17.8, M S_{\mathrm{e}}=23,596, p=.001\right]$. There was evidence for a main effect of format $\left[F(1,13)=4.4, M S_{\mathrm{e}}=\right.$ $124,080, p=.06]$ owing to a 94-msec RT advantage for small $\mathrm{S}-$ problems $(1,273 \mathrm{msec})$, as compared with $\mathrm{S}+$ $(1,367 \mathrm{msec})$. The format $\times$ appearance interaction was not significant $\left[F(1,13)=2.9, M S_{\mathrm{e}}=44,376, p=.11\right]$, but Table 2 shows that the evidence for an effect of format owed entirely to a $190-\mathrm{msec}$ advantage for small problems presented in the standard $\mathrm{S}-$ format (e.g., $9-6=\ldots$ ) as compared with the $\mathrm{S}+$ format $(9=6+\ldots)[t(13)=2.6$, $S E=72.0, p=.02]$. Thus, in contrast with large problems for which the addition format facilitated responding, performance on small subtractions was less efficient in the addition format than in the standard subtraction format.

The analysis of tie subtraction RTs indicated only an effect of position $\left[F(1,13)=5.7, M S_{\mathrm{e}}=6,967, p=.03\right]$, owing to a faster mean RT with the missing element on the right $(1,159 \mathrm{msec})$ than with it in the center $(1,212 \mathrm{msec})$, but no main or interaction effect involving format ( $p$ s > $.20)$.

Percentage of errors. Mean percentage of errors on subtraction problems (see Table 2) received a $2 \times 2 \times 3$ ANOVA with factors of format, problem type, and appearance. The ANOVA indicated only significant main effects.
As in Experiment 1, error rates for subtraction ties (3.8\%) and small problems $(2.5 \%)$ were low in comparison with those for large problems $(9.9 \%)\left[F(2,26)=17.1, M S_{\mathrm{e}}=\right.$ $51.3, p<.001]$. The error rate was slightly higher overall for the $\mathrm{S}+(6.1 \%)$ than for the $\mathrm{S}-(4.7 \%)$ format $[F(1,13)=$ $\left.6.2, M S_{\mathrm{e}}=12.6, p=.03\right]$. As Table 2 shows, operation errors (i.e., adding rather than subtracting the operands) were promoted by the $\mathrm{S}+$ format rather than the $\mathrm{S}-$. Finally, errors were less common with the missing element on the right $(4.6 \%)$ than with it in the center $(6.2 \%)[F(1,13)=8.2$, $\left.M S_{\mathrm{e}}=13.3, p=.01\right]$. The operation error data in Table 2 indicate that subtraction problems with the missing element in the standard position for subtraction (i.e., the right side) were less likely to erroneously trigger addition.

\section{Addition Problems}

RT. The mean RT for correct addition trials (see Table 2) received a $2 \times 3$ ANOVA with factors of format (subtraction, addition) and problem type (ties, small, large). Ties were answered fastest $(1,273 \mathrm{msec})$, followed by small $(1,529 \mathrm{msec})$ and large $(2,011 \mathrm{msec})$ problems $\left[F(2,26)=15.8, M S_{\mathrm{e}}=248,062, p<.001\right]$. As in Experiment 1 , addition performance was much faster in the $\mathrm{A}+$ format $(1,078 \mathrm{msec})$ than in the $\mathrm{A}-$ format $(2,130 \mathrm{msec})$

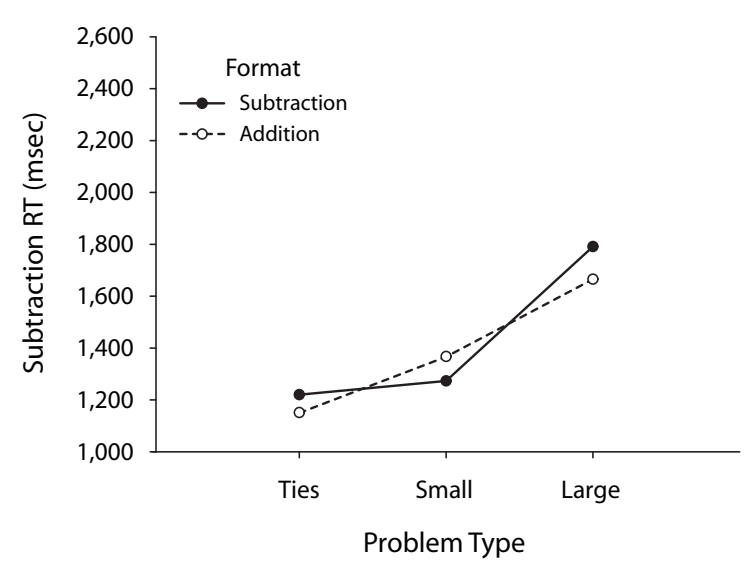

Figure 2. Mean subtraction response time (RT) as a function of problem type and format in Experiment 2. 
$\left[F(1,13)=39.1, M S_{\mathrm{e}}=594,103, p<.001\right]$, and again, this varied across problem type, with the A+ advantage greatest for large problems $(1,311 \mathrm{msec})$, followed by small $(1,056 \mathrm{msec})$ and then tie $(790 \mathrm{msec})$ problems $\left[F(2,26)=6.9, M S_{\mathrm{e}}=68,624, p=.004\right]$. For all three problem types, $\mathrm{A}+$ format $\left(7+6={ }_{-}\right)$yielded the fastest mean RT, as compared with any subtraction problem condition, whereas the $\mathrm{A}-$ format $(7=\ldots-6)$ required the longest RT on average. Thus, as in Experiment 1, participants found it particularly difficult to solve addition problems in subtraction format.

Percentage of errors. The corresponding analysis of addition errors (see Table 2) indicated only a main effect of problem type $\left[F(2,26)=16.8, M S_{\mathrm{e}}=65.8, p<.001\right]$ and of format $\left[F(1,13)=39.7, M S_{\mathrm{e}}=95.0, p<.001\right]$. Error rates for tie, small, and large subtraction problems were $5.8 \%, 7.4 \%$, and $17.4 \%$, respectively. Addition errors occurred less often with problems in the $\mathrm{A}+$ format $(3.5 \%)$ than with those in the $\mathrm{A}-$ format $(16.9 \%)$. Table 2 shows that the $\mathrm{A}-$ format $(7=\ldots-6)$ was the most errorprone condition, regardless of problem type, and that this owed largely to a high proportion of operation errors.

\section{Discussion}

Experiment 2 confirmed that large subtraction problems in the addition format $(13=6+\ldots)$ were solved more quickly than they were in the subtraction format $\left(13-6={ }_{-}\right)$. This result is expected if large subtractions were often mediated by addition, because the $\mathrm{S}+$ format would facilitate applying the addition reference strategy. The Experiment 2 RT advantage for large S+ problems as compared with the $\mathrm{S}$ - format was half that of Experiment 1 (125 msec vs. $254 \mathrm{msec})$. Part of the S+ advantage for large subtractions in Experiment 1 could be due to the minuend's appearing in the standard right-side position for addition (e.g., $6+_{-}=13$ ), whereas in Experiment 2, the $\mathrm{S}+$ format resembled subtraction with the minuend on the left (e.g., $13=6+\ldots$ ). This could make it relatively more difficult to access the relevant addition fact. It is important to consider, however, that participants in Experiment 2 overall solved large problems $502 \mathrm{msec}$ faster than did those in Experiment 1, whereas tie and small problems were solved only $95 \mathrm{msec}$ faster on average. It is possible that random sampling variability resulted in a slightly more arithmetically skilled group of participants in Experiment 2, as compared with Experiment 1. We would expect more skilled-related variability in RT for the larger, more difficult problems. Thus, participant differences might contribute to the reduced $\mathrm{S}+$ advantage for large problems in Experiment 2. Nonetheless, the RT advantage for large $\mathrm{S}+$ subtraction problems remained statistically robust with the $\mathrm{S}+$ format arranged to resemble subtraction, as in Experiment 2.

In contrast, small subtraction problems were answered more quickly in the $\mathrm{S}-$ format $\left(5-2=\_\right)$than in the $\mathrm{S}+$ format $(5=2+\ldots)$. If small subtractions were solved by direct retrieval (see LeFevre et al., 2006; Seyler et al., 2003), then the standard subtraction format probably would provide a better retrieval cue than the addition for- mat. Subtraction ties also are generally solved by direct retrieval according to self-reports (Campbell \& Gunter, 2002), but there was no RT advantage for ties in the Sformat $(12-6=\ldots)$ as compared with the $S+$ format $(12=6+\ldots)$. The fact that memory strength for ties is very high (Campbell \& Gunter, 2002) and that the correct answer is one of the presented operands apparently made either format an effective retrieval cue for the difference.

With respect to addition performance, as in Experiment $1, \mathrm{~A}+$ problems $\left(6+7=\_\right)$were the easiest problems, whereas $\mathrm{A}-$ problems $(7=\ldots-6)$ were the most difficult problems and prone to operation errors (e.g., respond "one" to $7=\ldots-6$ ). Thus, again, participants were lured toward subtraction by the A- stimuli. The A - format in Experiment 1 mimicked standard subtraction with the implied minuend on the left (e.g., _- $6=7$ ), whereas in Experiment 2, the layout did not mimic standard subtraction $\left(7={ }_{-}-6\right)$, which might have weakened the lure toward subtraction. Overall, the RT advantage for A+ as compared with $\mathrm{A}$ - problems was nominally smaller in Experiment $2(1,052 \mathrm{msec})$ than in Experiment 1 $(1,134 \mathrm{msec})$, and this trend was most pronounced for large addition problems (RT advantage of 1,446 msec in Experiment 1 vs. 1,331 msec in Experiment 2). Participant differences between experiments could have contributed to this, however, given that in Experiment 2, participants generally were faster than were those in Experiment 1, particularly on more difficult problems.

\section{GENERAL DISCUSSION}

Both experiments demonstrated that latency to solve basic subtraction problems depended on presentation format. Experiment 1 showed that subtractions with a minuend greater than 10 gained a substantial RT advantage when presented in addition format $\left(6+{ }_{-}=13\right.$ or - $+6=13)$ as compared with the subtraction format $\left(13-6={ }_{-}\right.$or $\left.13-_{-}=6\right)$. This effect remained robust in Experiment 2, with the addition-based format rearranged to resemble the standard subtraction format with the sum $/$ minuend on the left $(13=6+\ldots)$. The persistence of the addition format advantage with $\mathrm{S}+$ problems arranged to resemble subtraction reinforces the conclusion that the addition reference strategy is used for subtraction. Nonetheless, the effect was substantially smaller in Experiment $2(125 \mathrm{msec})$ than in Experiment $1(254 \mathrm{msec})$. This difference might be attributed to sampling variability, but nonetheless, it raises the possibility that part of the format effect on large subtractions in Experiment 1 was associated with the minuend's appearing in the standard right-side position for addition (e.g., _ $+6=13$ ). The latter arrangement could provide a better retrieval cue for addition fact retrieval or facilitate encoding to execute an addition procedure. Mauro et al. (2003) suggested that longer latencies given the standard format (for division in their study) reflected time taken to rearrange elements into the recast format (multiplication in their study). In this case, the smaller advantage in Experiment 2 for subtraction in addition format would occur because, with the 
sum and equal sign on the left (e.g., $13=6+\ldots$ ), the elements would need to be rearranged to correspond to standard addition with the sum and equal sign on the right. The RT advantage for subtraction in the addition format in Experiment 2 presumably occurred because the plus sign provided a better retrieval cue for the mediating addition fact - or provided a more effective cue for an addition procedure - than did the subtraction format. The presentation of the addition plus sign appears to be sufficient to enhance mediation and speed RT for large subtractions.

In contrast, neither ties nor small subtraction problems presented an RT advantage in addition format. This was expected, because ties and small subtractions are believed to be solved predominantly by direct retrieval and not mediated by addition (Campbell \& Gunter, 2002; Campbell $\&$ Xue, 2001). Consequently, facilitated access to the corresponding addition fact would not be expected to benefit performance of the tie and small subtractions. Indeed, in Experiment 2, small subtractions were solved more slowly in the addition than in the subtraction format. The standard subtraction format (e.g., $5-2=\_$) apparently provided a better retrieval cue than did the unfamiliar addition format $(5=2+\ldots)$.

Subtraction performance was also affected by appearance, which was manipulated via the location of the missing element in the presented problem. In both experiments, latencies were faster with subtraction problems in the standard format (e.g., $13-6=\ldots$ ) as compared with an unusual format (e.g., $13-{ }_{-}=6$ ). This result suggests that problem encoding routines for simple arithmetic are specialized for the standard format and that when presented with an unusual layout, participants must reorganize or conceptually reassign numerical components in order to solve the problem (e.g., conceptually reassign 6 from the answer to the subtrahend to solve $13-_{-}=6$ ).

In both experiments, addition in addition format (e.g., $\left.6+7={ }_{-}\right)$was the fastest and most accurate condition for tie, small, and large problems. Taken together with the evidence that addition mediates subtraction, the speed and accuracy of addition affirms it as the basic operation and subtraction as the subordinate operation. Addition in the addition format was the easiest; conversely, addition in the subtraction format (e.g., ${ }_{-}-6=7$ ) was the most difficult condition both in latency and in error rate, especially for large problems. There likely are multiple factors underlying the difficulty of addition in subtraction format. First, ties (e.g., ${ }_{-}-2=2$ ) and small $\left({ }_{-}-2=3\right.$ ) addition problems in subtraction format could be mediated by retrieval of the corresponding subtraction fact, or recast as addition, which would inflate RT relative to standard addition format. Large addition problems in subtraction format would rarely, if ever, be solved by the retrieval of the subtraction counterpart and would need to be recast as addition and then solved by retrieval or procedure, incurring substantial RT costs. Moreover, two thirds of trials involved subtraction, which would create a subtraction bias. Combined with the minus sign in subtraction format addition problems, the subtraction bias would make it difficult for participants to decide between addition and subtraction, slowing RT and promoting operation errors (e.g., responding "one" instead of "thirteen" to _- $6=7$ ).

\section{Relation to Previous Research}

The present findings converge with previous research in which participants' self-reported strategies identified addition reference as a common subtraction strategy, especially for large subtractions (LeFevre et al., 2006; Seyler et al., 2003). In both of the present experiments, performance on large, but not on small or tie, subtractions was facilitated by the addition format, which would be expected if large subtractions were solved using the addition reference strategy. In contrast, Campbell et al. (2006) failed to find evidence that practicing a specific addition prime $(6+7)$ facilitated RT on the corresponding subtraction probe $(13-7)$ tested a few trials later. If mediation occurred, we would normally expect recent practice of the mediator to speed up mediation, but this did not occur. This seems to be difficult to reconcile with the evidence for mediation on the basis of participants' self-reports and the present experiments. Campbell et al. also found, however, that reported direct retrieval for subtraction increased significantly $(+2.3 \%)$ following recent retrieval of the corresponding addition fact. We would not expect subtraction to mediate addition; consequently, mediation is an unlikely explanation for this effect. One possibility is that this effect reflects covert practice of the subtraction counterpart during the addition prime trial. Given that the inverse addition and subtraction problems (e.g., $8+6,14-8)$ were often tested a few trials apart in the Campbell et al. paradigm, it would be prudent for participants to think about the more difficult subtraction counterpart problem between trials, in anticipation that it would be tested soon. Covert practice of subtraction on the addition prime trial could promote attempts at direct retrieval for the subsequent subtraction probe and work against evidence for mediation. If this is correct, then the Campbell et al. experiment would have provided an insensitive measure of mediation.

Rickard (2005) provided evidence that memory for multiplication facts entails a reverse association from product to factors, which would facilitate mediation of division by multiplication (see also Campbell \& Robert, 2008; Rusconi et al., 2006). Rickard noted, however, that a reverse association for addition (i.e., a direct association from sum to addends) would not develop because, unlike most products, sums do not correspond to a unique pair of operands. If there is no reverse association for addition, how is the addition reference strategy for subtraction accomplished? Although a sum (e.g., 13) does not specify a unique pair of addends, a subtraction problem (e.g., 13 - 6) does specify a unique addition problem $(6+7=13)$. With respect to the addition reference strategy, the subtraction stimulus thereby provides the sum and one addend, which serve as a compound retrieval cue for the addition counterpart. Presenting the subtraction problem in addition format facilitates this process by reducing the internal manipulations required to obtain an effective retrieval cue for the corresponding addition fact. The RT 
advantage for large subtractions in addition format as compared with standard subtraction format was much larger (254 msec and $125 \mathrm{msec}$ in Experiments 1 and 2, respectively) than the 66-msec RT advantage observed for division in multiplication format by Mauro et al. (2003). The availability of the reverse multiplication association to mediate division would minimize the internal manipulations required to perform division by multiplication reference; consequently, presenting the problem in multiplication format would provide relatively modest gains.

\section{Conclusions}

In two experiments, we tested the hypothesis that educated adults often solve large simple subtraction problems by reference to the corresponding addition fact but that they rely on direct memory retrieval for ties and small subtractions. In both experiments, participants were substantially faster to solve large subtractions presented in addition format, but there were no gains with addition format for either ties or small subtractions. In fact, in Experiment 2 , small subtractions were solved faster in standard format than in addition format. These findings provide strong converging evidence that educated adults often use an addition reference strategy to solve large subtractions but that they use direct retrieval for ties and small subtractions (LeFevre et al., 2006; Seyler et al., 2003). More generally, the results add to the evidence that educated adults do not rely exclusively on direct memory retrieval for basic arithmetic, but instead use a variety of procedural strategies, especially for large problems. Procedures - such as the addition reference strategy for subtraction or the multiplication reference strategy for division - apparently constitute an essential core component of skilled adults' arithmetic repertoire (cf. Campbell \& Xue, 2001).

\section{AUTHOR NOTE}

This research was supported by a grant from the Natural Sciences and Engineering Research Council of Canada. The author thanks Rich Carlson and two anonymous reviewers for very helpful feedback on a previous version of the manuscript. Address correspondence to J. I. D. Campbell, Department of Psychology, University of Saskatchewan, 9 Campus Drive, Saskatoon, SK, S7N 5 A5 Canada (e-mail: jamie .campbell@usask.ca).

\section{REFERENCES}

AshCraft, M. H. (1992). Cognitive arithmetic: A review of data and theory. Cognition, 44, 75-106.

AshCRAFT, M. H. (1995). Cognitive psychology and simple arithmetic: A review and summary of new directions. Mathematical Cognition, 1, 3-34.

Barrouillet, P., Mignon, M., \& Thevenot, C. (2008). Strategies in subtraction problem solving in children. Journal of Experimental Child Psychology, 99, 233-251.

Campbell, J. I. D. (1995). Mechanisms of simple addition and multiplication: A modified network-interference theory and simulation. Mathematical Cognition, 1, 121-164.

CAMPBELL, J. I. D. (1997). Reading-based interference in cognitive arithmetic. Canadian Journal of Experimental Psychology, 51, 74-81.

CAmpbell, J. I. D. (1999). Division by multiplication. Memory \& Cognition, 27, 791-802.
CAmpbell, J. I. D. (ED.) (2005). Handbook of mathematical cognition. New York: Psychology Press.

Campbell, J. I. D., Fuchs-Lacelle, S., \& Phenix, T. L. (2006). Identical elements model of arithmetic memory: Extension to addition and subtraction. Memory \& Cognition, 34, 633-647.

Campbell, J. I. D., \& Graham, D. J. (1985). Mental multiplication skill: Structure, process, and acquisition. Canadian Journal of Psychology, 39, 338-366.

CAmpbell, J. I. D., \& Gunter, R. (2002). Calculation, culture, and the repeated operand effect. Cognition, 86, 71-96.

CAmpbell, J. I. D., \& RoberT, N. D. (2008). Bidirectional associations in multiplication memory: Conditions of negative and positive transfer. Journal of Experiment Psychology: Learning, Memory, \& Cognition, 34, 546-555.

CAmpbell, J. I. D., \& Timm, J. C. (2000). Adults' strategy choices for simple addition: Effects of retrieval interference. Psychonomic Bulletin \& Review, 7, 692-699.

Campbell, J. I. D., \& Xue, Q. (2001). Cognitive arithmetic across cultures. Journal of Experimental Psychology: General, 130, 299-315.

Geary, D. C., Frensch, P. A., \& Wiley, J. G. (1993). Simple and complex mental subtraction: Strategy choice and speed-of-processing differences in younger and older adults. Psychology \& Aging, 8, $242-256$.

Geary, D. C., \& Wiley, J. G. (1991). Cognitive addition: Strategy choice and speed-of-processing differences in young and elderly adults. Psychology \& Aging, 6, 474-483.

Неснт, S. A. (1999). Individual solution processes while solving addition and multiplication math facts in adults. Memory \& Cognition, 27, 1097-1107.

LeFevre, J., Bisanz, J., Daley, K. E., Buffone, L., Greenham, S. L., \& SADESKY, G. S. (1996). Multiple routes to solution of single-digit multiplication problems. Journal of Experimental Psychology: General, 125, 284-306.

LeFevre, J., DeStefano, D., Penner-Wilger, M., \& Daley, K. E. (2006). Selection of procedures in mental subtraction. Canadian Journal of Experimental Psychology, 60, 209-220.

LeFevre, J., \& Morris, J. (1999). More on the relation between division and multiplication in simple arithmetic: Evidence for mediation of division solutions via multiplication. Memory \& Cognition, 27, 803-812.

LeFevre, J., Sadesky, G. S., \& Bisanz, J. (1996). Selection of procedures in mental addition: Reassessing the problem size effect in adults. Journal of Experimental Psychology: Learning, Memory, \& Cognition, 22, 216-230.

Mauro, D. G., LeFevre, J., \& Morris, J. (2003). Effects of problem format on division and multiplication performance: Division facts are mediated via multiplication-based representations. Journal of Experimental Psychology: Learning, Memory, \& Cognition, 29, 163-170.

RICKARD, T. C. (2005). A revised identical elements model of arithmetic fact representation. Journal of Experimental Psychology: Learning, Memory, \& Cognition, 31, 250-257.

Robinson, K. M. (2001). The validity of verbal reports in children's subtraction. Journal of Educational Psychology, 93, 211-222.

Rusconi, E., Galfano, G., Rebonato, E., \& Umiltà, C. (2006). Bidirectional links in the network of multiplication facts. Psychological Research, 70, 32-42.

Seyler, D. J., Kirk, E. P., \& Ashcraft, M. H. (2003). Elementary subtraction. Journal of Experimental Psychology: Learning, Memory, \& Cognition, 29, 1339-1352.

Siegler, R. S., \& Shrager, J. (1984). A model of strategy choice. In C. Sophian (Ed.), Origins of cognitive skills (pp. 229-293). Hillsdale, NJ: Erlbaum.

Zbrodoff, N. J., \& Logan, G. D. (2005). What everyone finds: The problem size effect. In J. I. D. Campbell (Ed.), Handbook of mathematical cognition (pp. 331-346). New York: Psychology Press.

(Manuscript received February 23, 2008; revision accepted for publication April 21, 2008.) 